\title{
Local ionospheric electrodynamics associated with neutral wind fields at low latitudes: Kelvin-Helmholtz billows
}

\author{
J. P. St.-Maurice and R. K. Choudhary \\ Institute of Space and Atmospheric Studies, University of Saskatchewan, Saskatoon, Canada \\ Received: 28 September 2005 - Revised: 20 January 2006 - Accepted: 28 March 2006 - Published: 3 July 2006 \\ Part of Special Issue "The 11th International Symposium on Equatorial Aeronomy (ISEA-11), Taipei, May 2005”
}

\begin{abstract}
The Gadanki radar observation of plasma irregularities bearing the signature of Kelvin-Helmholtz billows above $100 \mathrm{~km}$ altitude raises the question of the electrodynamical mechanism that would allow the structures to drift with the neutral wind. We show that for locally varying neutral wind fields with the right geometry at night, multiple Hall effects in the electron gas lead to a situation where ions, electrons, and neutrals move together along the component of the wind that changes most rapidly in space. The species must not move together along all directions, however. If this were the case the plasma would be stable and a radar would be unable to observe the wind field. We discuss the stability of the plasma itself for Es layers affected by the Kelvin-Helmholtz wind field and show that a variety of factors have to be taken into account beyond the study of the zeroth order mechanism.
\end{abstract}

Keywords. Ionosphere (Equatorial ionosphere; Ionospheric irregularities; Plasma waves and instabilities) - Meteorology and atmospheric dynamics (Turbulence)

\section{Introduction}

Choudhary et al. (2005) have recently reported on the observations of tilted layers of $3 \mathrm{~m}$ irregularities near $100 \mathrm{~km}$ altitude using the Gadanki radar which is located in India at $13.5^{\circ}$ North, $79.2^{\circ}$ East (geographic), $6.4^{\circ}$ North of the geomagnetic equator. In the cleanest examples that they presented, flow reversals were found near the altitude center of the echoing region, while the Doppler shift would otherwise gradually change sign from the bottom-side echo region to the topside along a tilt. These properties were shown to be the signature of classic Kelvin-Helmholtz (KH) billows. We should note that, before reaching this conclusion, Choud-

Correspondence to: J. P. St.-Maurice

(jp.stmaurice@usask.ca) hary et al. (2005) looked at various mechanisms that could have generated the particular QP echoes that they observed. These included atmospheric gravity waves (Woodman et al., 1991)(periods too small, requiring too large a Doppler shift), and the Cosgrove-Tsunoda mechanism (Cosgrove and Tsunoda, 2002) (latitude too small, with magnetic field not tilted enough). Reasonable shears could furthermore be inferred from the observations, implying Richardson numbers that were large enough to make $\mathrm{KH}$ billows a plausible explanation while agreeing with the gist of the Larsen (2000) observations. The tilted echo layers were argued to move horizontally through the radar field of view while the geometry of the observation and the beam pattern were such that there was no range-height ambivalence, in contrast with QP observations made at higher latitudes than Gadanki. Finally, the data with clean echo patterns had to be associated with $\mathrm{KH}$ billows in their early stages of evolution, in qualitative agreement with the patterns produced in numerical simulations by Bernhardt (2002).

In the Choudhary et al. (2005) presentation the emphasis was placed on showing how the inferred wind field was a good match to expectations from a $\mathrm{KH}$ billow in its early stages of evolution. There was only a rough outline of the electro-dynamical mechanism that would lead to the production of $3 \mathrm{~m}$ irregularities inside Kelvin-Helmholtz billows. This question nevertheless remains an important part of the puzzle. While the observations are showing that the wind field is that of a $\mathrm{KH}$ billow, the question that remains to be investigated in more detail is: how can the Doppler shift of irregularities, supposed to respond only to an electron $\boldsymbol{E} \times \boldsymbol{B}$ drift, reproduce the vertical neutral wind pattern in the $\mathrm{KH}$ billows when, at first sight, the electrons should not respond to the wind? The question of irregularity generation under $\mathrm{KH}$ billow conditions also needs to be addressed.

Our aim here is therefore to provide a more precise description of the local electrodynamics that leads to the production of m-size irregularities. For clarity, we limit our

Published by Copernicus GmbH on behalf of the European Geosciences Union. 


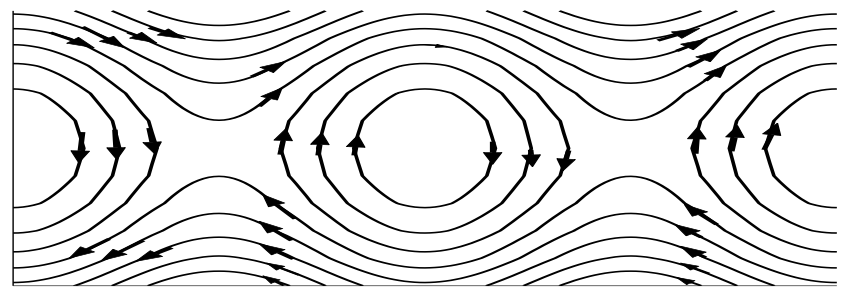

Fig. 1. Contour plot of the stream function associated with KelvinHelmholtz billows. The vertical and horizontal distances respectively represent the extent of the vertical $(\sim 6 \mathrm{~km})$ and horizontal $(\sim 16 \mathrm{~km})$ displacements inside the billow.

discussion to the $\mathrm{KH}$ wind field mechanism at low latitudes at night. However, it should become clear from the presentation that similar processes could operate under various divergent/convergent horizontal or vertical wind fields on more complicated field line geometries, particularly at night.

The presentation that follows has two main parts. First we discuss the electrodynamics per se under idealized geometrical conditions that nevertheless should provide a good description of the $\mathrm{KH}$ billows at low latitudes. Having discussed the electrodynamics we then address the question of the ensuing generation of the irregularities and their eventual detection by a radar. We then explore briefly the possible effects of complications arising in more realistic situations than the zeroth order one that we have focused on.

\section{Basic model}

\subsection{Es layers and their distortions}

Away from the magnetic equator itself it is becoming clear that the observation of $\mathrm{m}$-size irregularities with radars is associated with the presence of sporadic Es layers that provide the necessary gradients and localized electric fields to excited gradient-drift instabilities (Haldoupis and Schlegel, 1996; Hussey et al., 1998). At low latitudes and well below $120 \mathrm{~km}$, as is the case for the Gadanki echoes under consideration here, the creation of an Es layer requires in the first place that there be a strong shear in the zonal wind (Whitehead, 1970). Since a westward wind lifts the ions and an eastward wind lowers them, the creation of the layer requires the lower zonal wind to move west and the upper zonal wind to move east. As long as the $\mathbf{B}$ lines are not strictly horizontal over the sheared region, this mechanism does not create an electric field; the negative charges left behind when the ions are ascending become reconnected to positive charges higher up, through a $\mathbf{B}$ line that passes through both the top and bottom of the Es layer.

Under strong enough shear conditions, the zonal wind shears that create the Es layer may also be KH unstable. In their simplest linear local manifestation (Kundu, 1990) the billows involve a superposition of the original zonal wind shears with a simple sinusoidal oscillation in the vertical wind, that is, a vertical wind of the form $V_{v} \sin (k x)$ where $x$ refers, in this case, to the zonal direction. When this is the case, we argue here that the combination of the zonal and vertical wind fields, and of the Es layer, implies that short scale plasma waves will be moving with the vertical neutral wind in the magnetic northward direction (almost the vertical at low latitudes).

In what follows we construct a simple electro-dynamical model of the plasma below $110 \mathrm{~km}$ in the presence of "weakly-excited" KH billows. By "weakly-excited", we mean billows that are in their early stages of evolution so that turbulence has not yet evolved to the point of either destroying the original Es layers and/or of making the layer unrecognizable with radar Doppler measurements. Our model is anchored on the simulations done by Bernhardt (2002), as shown in his Figs. 6 (panels $b$ and c) and 7 (panels a and b). These figures clearly show that, starting roughly $100 \mathrm{~s}$ after the onset and lasting for several hundred seconds afterwards, the Es layer is tilted - as expected from weakly excited situations- by the vertical wind field that triggers the billows. The orientation of the tilts is dictated by the sign of the zonal wind shears. The angle of the tilt grows with time while, at the same time, the Es layer thins out. While the thinning is taking place, the original Es layer evolves to the point of splitting into two new layers placed at the top and bottom parts of the unstable KH billow region. Bernhardt (2002) obtains this evolution in the lower E region, below $110 \mathrm{~km}$. Near $120 \mathrm{~km}$, the thinning out the Es layer and its splitting into top and bottom layers do not take place. Instead, he obtains, after a while, stable tilted "islands" of ionization in the middle of the KH billows. Either way, tilts of ionization are common for much of the simulation time. We should also note that if 3-D simulations were used, turbulence would likely ultimately destroy the original structures (Gibson-Wilde et al., 2000).

\subsection{Magnetic field orientation}

The simplest model that we can construct to study the $\mathrm{KH}$ instability problem in the present context starts with the following: since we are interested in very low latitudes we will neglect the tilt from the horizontal in the magnetic field lines. This is not to say that the tilt is not important in eliminating perpendicular northward-vertical electric fields that would otherwise limit the growth of the Es layer. Our neglect of the tilt is simply so as to clarify the mechanism at work: introducing weak tilts in the basic model does not add much to the calculated effects.

\subsection{Neutral wind model}

In accordance with the early stages of evolution of the $\mathrm{KH}$ billows and consistent with the formation of an Es layer below $110 \mathrm{~km}$, we introduce the following possible neutral 
wind configuration: we use a simple zonal wind shear of the kind required to produce an Es layer in the first place. In the region of interest, this zonal wind field is assumed to change only with altitude, that is, not with latitude or longitude. To this basic wind field we add a $V_{v} \sin (k x)$ vertical wind, with $x$ now explicitly referring to the west-east direction. A third element, namely, a background zonal wind, will also be required for the purpose of explaining how a northwardvertical looking radar can see the passage of various billows as a function of time.

The simple "cat's eyes" flow pattern corresponding to our wind pattern is shown in Fig. 1, in the frame of reference of the mean wind. That is to say, the figure depicts the streamlines associated with the presence of a linear shear in the zonal wind, superposed with the simple oscillating vertical wind field mentioned above. In this particular context, the wind rolls have an "infinite" long north-south extent (through the paper). This simply means here that the north-south extent is very long in comparison to the zonal wavelength associated with oscillations in the vertical wind.

\subsection{Electro-dynamical assumptions}

In modeling the plasma response to the wind field we will (1) neglect chemistry, (2) neglect pressure gradients and gravity, (3) assume a quasi-steady state, (4) neglect parallel electric fields, and (5) assume that the conductivities are dominated by the local Es layer. We now discuss the validity of these assumptions.

Assumption (1): We are dealing here with nighttime situations in which shears in the neutral wind causes the concentration to increase in a thin layer. Clearly, if the layer is still present in spite or recombination and in the absence of photo-production, this implies that metals must dominate the ion population. The recombination of metallic ions is extremely slow above $90 \mathrm{~km}$ (Riggin et al., 1986).

Assumption (2): The best that perpendicular pressure gradients and gravity can do is induce $\boldsymbol{F} \times \boldsymbol{B}$ drifts, where $\mathbf{F}$ is either the pressure or the gravitational force. In the case of gravity, the resulting drifts even for magnetized ions, would be of the order of $\mathrm{cm} / \mathrm{s}$. For electrons, the drift is orders of magnitude less. Similarly, the diamagnetic drifts should be of the order of $500 \nabla \ln n_{0} \mathrm{~m} / \mathrm{s}$ for both ions and electrons, assuming a temperature of the order of $300 \mathrm{~K}$. Even for $100 \mathrm{~m}$ gradient scales this implies drifts of the order of $5 \mathrm{~m} / \mathrm{s}$, which is less than the drifts under consideration here. Finally, since we assume the region to be elongated along the magnetic field, ordinary pressure gradients will not have an effect in that direction either.

Assumption (3): We assume a steady state. We do not mean by this that the KH billows do not evolve with time. We mean rather that the $\mathrm{KH}$ evolution is much slower than the electrodynamical response of the plasma. When electric fields are formed by the neutral winds, the electron response time to the new field is at most equal to the electron collision frequency time. This is equivalent to saying that we drop the term $\partial \rho / \partial t$ in the current continuity equation.

Assumption (4): We use the non-zero tilt in the magnetic field to let the Es layer remain neutral, just as the classical mechanism does. The zonal wind shears push the ions into the layer but electrons are left behind. However, the positive ion layer is connected to the negative electrons left behind via the inclined magnetic field lines. The electron response is very fast, leaving only a very small parallel electric field behind. A small parallel electric field remnant is also left from the edges of the wind field, which we therefore have to assume are far apart.

Assumption (5): The $110 \mathrm{~km}$ altitude region at Gadanki is connected to the equatorial region at $180 \mathrm{~km}$ through the magnetic field line. This altitude region is too low for ionization to survive recombination at night (too much molecular nitrogen). This means that the lower Gadanki E region is not connected to an F region in which Pedersen conductivities would be enhanced, which would allow perpendicular currents to be discharged via the $\mathrm{F}$ region through the mediation of parallel currents between the two. We can therefore assume that the electrodynamics is entirely a local affair, in that context. There remains one last possibility for nonlocal effects, namely, the existence of a conjugate $\mathrm{E}$ region on the other side of the magnetic equator. However, the distance between the two $\mathrm{E}$ regions is small enough that we normally have nighttime conditions in both places. This means that both $\mathrm{E}$ regions would have to be subject to Es layers simultaneously. If this were the case, there is no question that our simple description would not apply. By the same token, however, the likelihood that simple wind patterns would be observed at all in the presence of conjugate Es layers (particularly broken ones) is remote to none.

\section{Physical mechanism}

\subsection{Basic description}

We are now ready to consider the electrodynamical effects introduced by the vertical wind for the geometry at hands. Before considering the attendant equations, let us first describe the basics of the mechanism at hands: to leading order, since the ions are highly collisional, they are pushed up and down by the oscillations in the vertical wind over long distances along the north-south direction. By contrast, the electrons remain strongly magnetized and do not directly respond to the wind. The vertical wind therefore introduces a current. However, if there is any divergence in the wind, that is, specifically, if the vertical wind extends over a finite altitude range, the vertical currents have to diverge at first, until a charge separation between ions and electrons is introduced. After a short time the ensuing electric field has become large enough to eliminate the current divergence along the vertical direction. One way for this to happen would be to have 


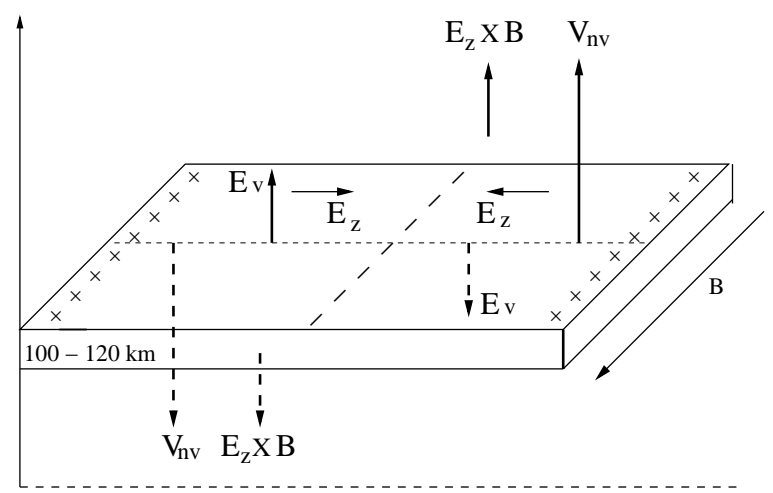

Fig. 2. Schematic diagram showing the effect of vertical wind on sporadic-E ion layer. Here $\mathrm{V}_{n v}$ represents the vertical wind. $\mathrm{E}_{z}$ and $\mathrm{E}_{v}$ are zonal and vertical fields respectively

an electric field such that the resulting ion Pedersen current would produce an ion motion equal and opposite to the motion produced by the neutral wind.

However, the introduction of a new spatially oscillating vertical electric field in the system means that Hall currents are introduced by the $\boldsymbol{E} \times \boldsymbol{B}$ motion of the electrons in the east-west or west-east directions. The situation is depicted in the schematic diagram shown in Fig. 2. Owing to the elongation in the north-south direction, a spatially oscillating electric field is now introduced in the east-west direction by the accumulations of electrons at every second vertical wind node. The horizontal electric field increases until the net horizontal force on the electrons becomes zero, a classical Hall effect.

In turn, the new oscillating horizontal electric field introduces a vertical electron Hall drift. This second Hall effect now moves electrons in the same direction as the oscillating vertical wind. Therefore the electrons are now flowing vertically in the same direction as the ions. This brings up the question of closure: the electrons have to move at the exact same speed as the ions. If they don't, the vertical Hall currents will lead to a different vertical charge arrangement and a different vertical field, which in the end will modify the vertical currents once more. Also, since the ions are moving at close to the neutral wind velocity, so will the electrons. The plasma, in other words, will move up and down with the neutral wind in $\mathrm{KH}$ billows of the sort described here.

\subsection{Quantitative description}

Since our focus is on motions perpendicular to the magnetic field direction, we neglect the small pressure gradient effects and gravity for the reasons given at the beginning of this section. In that case, the steady state ion equation of motion in the collisional ionosphere can be written as

$$
v_{i}\left(\mathbf{V}_{i}-\mathbf{V}_{n}\right)=\Omega_{i} \frac{\mathbf{E}}{B}+\Omega_{i} \mathbf{V}_{i} \times \mathbf{b}
$$

where $\mathbf{V}_{i}$ is the mean ion velocity, and $\Omega_{i}$ and $v_{i}$ are the ion cyclotron and ion-neutral collision frequencies respectively, while $B$ is the magnetic field strength and $\mathbf{b}$ is a unit vector in the magnetic field direction. Using $\alpha_{i}=\Omega_{i} / \nu_{i}$, and assuming the electric field to be perpendicular to the geomagnetic field because of the high parallel conductivities even in the E region, we can write the vector solution to the momentum balance as

$$
\begin{aligned}
& \mathbf{V}_{i}=\mathbf{V}_{n \|}+\frac{\mathbf{V}_{n \perp}}{1+\alpha_{i}^{2}}+\frac{\alpha_{i}}{1+\alpha_{i}^{2}} \mathbf{V}_{n \perp} \times \mathbf{b} \\
& +\frac{\alpha_{i}}{1+\alpha_{i}^{2}} \frac{\mathbf{E}}{B}+\frac{\alpha_{i}^{2}}{1+\alpha_{i}^{2}} \frac{\mathbf{E} \times \mathbf{b}}{B}
\end{aligned}
$$

where $\mathbf{V}_{n \|}$ and $\mathbf{V}_{n \perp}$ are the neutral wind components parallel and perpendicular to the geomagnetic field, respectively.

A similar solution is obtained for the electrons except for the fact that the charge changes sign. As a result, if we define $\Omega_{e}$ and $\alpha_{e}$ as positive, we obtain

$$
\begin{aligned}
& \mathbf{V}_{e}=\mathbf{V}_{n \|}+\frac{\mathbf{V}_{n \perp}}{1+\alpha_{e}^{2}}-\frac{\alpha_{e}}{1+\alpha_{e}^{2}} \mathbf{V}_{n \perp} \times \mathbf{b} \\
& -\frac{\alpha_{e}}{1+\alpha_{e}^{2}} \frac{\mathbf{E}}{B}+\frac{\alpha_{e}^{2}}{1+\alpha_{e}^{2}} \frac{\mathbf{E} \times \mathbf{b}}{B} .
\end{aligned}
$$

With Eqs. (2) and (3) we can obtain the currents, which are proportional to $\left(\mathbf{V}_{i}-\mathbf{V}_{e}\right)$, which is itself given by

$$
\begin{aligned}
& \left(\mathbf{V}_{i}-\mathbf{V}_{e}\right)=\frac{\mathbf{E}^{\prime}}{B}\left[\frac{\alpha_{i}}{1+\alpha_{i}^{2}}+\frac{\alpha_{e}}{1+\alpha_{e}^{2}}\right] \\
& +\frac{\mathbf{E}^{\prime} \times \mathbf{b}}{B}\left[\frac{1}{1+\alpha_{e}^{2}}-\frac{1}{1+\alpha_{i}^{2}}\right]
\end{aligned}
$$

where

$\mathbf{E}^{\prime}=\mathbf{E}+\mathbf{V}_{n} \times \mathbf{B}$.

Next, we assume nightime conditions under which the only conducting region is the Es layer under observation: as discussed at the beginning of the section, if their is no conjugate Es layer, our nighttime Es layer is connected through the magnetic field to regions that are everywhere else poorly conducting because of their small electron densities. Under these conditions the currents induced by a vertical neutral wind have nowhere to close so that we should have $\mathbf{V}_{i v} \approx \mathbf{V}_{e v}$. Here the subscript $v$ refers to the "vertical" (strictly speaking, this should be the magnetically northward line-of-sight direction, which is near the vertical at low latitudes). As a result we find

$\mathbf{E}_{v}^{\prime}=\mathbf{E}_{z}^{\prime} \times \mathbf{b} \frac{\alpha_{e}-\alpha_{i}}{1+\alpha_{i} \alpha_{e}} \approx \mathbf{E}_{z}^{\prime} \times \mathbf{b} \frac{v_{i} / \Omega_{i}}{1+v_{e} v_{i} / \Omega_{e} \Omega_{i}}$. 
For the situation depicted in Fig. 2 the neutral wind oscillations are along the vertical. To a first approximation, we therefore end up with

$\mathbf{E}_{v}=\frac{v_{i} / \Omega_{i}}{1+v_{e} v_{i} / \Omega_{e} \Omega_{i}}\left(\mathbf{E}_{z} \times \mathbf{b}-\mathbf{V}_{n v} B\right)$

This result is consistent with the cartoon drawn in Fig. 2 in that the vertical field points in a direction opposite to the vertical neutral wind. However, the vertical field also clearly depends on the presence of a zonal electric field.

Repeating the calculation for zonal currents we end up with

$\mathbf{V}_{i z}-\mathbf{V}_{e z}=\frac{\mathbf{E}_{z}^{\prime}}{B}\left[\frac{\alpha_{i}}{1+\alpha_{i}^{2}}+\frac{\alpha_{e}}{1+\alpha_{e}^{2}}\right]$

$+\frac{\mathbf{E}_{v} \times \mathbf{b}}{B}\left[\frac{1}{1+\alpha_{e}^{2}}-\frac{1}{1+\alpha_{i}^{2}}\right]$

With result Eq. (7), this means that the zonal currents are proportional to $\mathbf{E}_{z}^{\prime}$. However, if zonal currents have nowhere to close (requires exactly the same conditions as for the "vertical" currents case), we end up requiring that $\mathbf{E}_{z}^{\prime}$ itself has to vanish.

The implication is that the solution to this system, to leading order, is simply given by $\mathbf{E}_{v} \approx 0$ and

$\mathbf{E}_{z} \approx-\mathbf{V}_{n v \perp} \times \mathbf{B}$.

Thus, under the reasonable assumption of vanishing conductivities outside the Es layer itself, all signs of the original vertical electric field would in fact be gone. To leading order, we therefore end up with a one-dimensional system where there is only a zonal electric field component. That component is such as to allow the electrons to move with the ions and the neutrals along the vertical direction. We remind the reader, however, that we have used a superposition principle here and that currents need not vanish in response, for example, to ambient large scale electric fields or to large scale zonal winds. Small shorting currents outside the Es layer region might also play a second order role.

\section{Irregularities and their properties}

Kagan and Kelley (1998) have discussed an instability mechanism for gradient-drift waves in the $\mathrm{E}$ region that works particularly well in the case presented here, that is, in a case for which zonal wind shears generate Es layers when the magnetic field is almost horizontal. As is well-known, gradientdrift (GD) waves are unstable in the E region when an electric field is perpendicular to the $\mathbf{B}$ field and the ambient electron density gradient has a component parallel to the electric field. However, Kagan and Kelley (1998) also correctly pointed out that a $\mathbf{V}_{n} \times \mathbf{B}$ field plays the same role as an electric field as the destabilizing agent. In this particular case the ions, rather than the electrons, are responsible for polarization electric fields, since ions are dragged by the neutral wind.

What is particularly interesting when an Es layer is produced by zonal neutral wind shears is that both sides of the Es layer are GD unstable since the zonal wind field reverses direction in just the right manner. Thus, near the nodes of the vertical neutral wind, we should expect both sides of the layer to be unstable for wavevectors pointing in the zonal direction. However, when the center of an Es layer has moved out of its equilibrium position, there would of course come a point when only one side is unstable, at least in the local context. Thus, if the layer moves upward, the top part remains unstable while the bottom part may become stable. The outcome obviously depends on the height shift and on the thickness of the layer. It remains nevertheless possible that both sides could remain unstable in such a case through 'nonlocal' effects. With one side being more unstable than the other and with only weak parallel electric fields through the layer, the tendency for growth on one side might be enough to stimulate growth on both sides (Seyler et al., 2004).

Whether or not both sides of the Es layer are unstable to GD waves in any given situation, the key point is that the most unstable wavevector direction for the GD instability is in the same direction as the zonal neutral wind that destabilizes the Es layer. This direction is perpendicular to the ambient electron density gradient, at least for the simplest cases. If the gradients were steep enough and the winds large enough, m-scale structures could be generated directly by the GD process; large amplitude m-scale structures observed by radars along the unstable direction are simply called "Type I". These structures may or may not be excited in our case. It seems more likely, however, that only larger structures would be directly excited by the GD process. Unlike m-size structures, the larger scale structures are not affected by the stabilizing influence of diffusion.

Whether or not m-size waves are excited directly in the zonal direction, there will be no growth from the linear instability mechanism along the direction of the density gradient. Meter-scale large amplitude waves are nevertheless observed in these directions. The structures are attributed to modecoupling triggered by turbulence. Their spectra, described as "Type II", are typically broader and less powerful than the Type I counterpart. That is to say, the energy not only cascades from 100-m size or km-size GD structures down to $\mathrm{m}$-size irregularities, but it also introduces large amplitude modes in the linearly stable direction through mode-coupling (Fejer, 1996).

According to observations and weak turbulence theory, the Doppler shift of Type II waves matches the value expected from linear theory (though the linear modes are themselves damped) (Kelley, 1989). In the situation of interest in the present work, for an observer looking up (ie perpendicular to the direction of maximum growth), the Doppler shift should therefore normally be zero (zero frequency modes). However, in the special case for which our argument that both 
ions and electrons are moving together with the vertical neutral wind, the implication is that the Doppler shift of Type II structures will match the vertical neutral wind velocity. This result satisfies the usual expressions for the Doppler shift. Specifically, for Type II waves, the Doppler shift is normally taken to be

$\mathbf{V}_{p h}=\hat{\mathbf{k}} \hat{\mathbf{k}} \cdot \frac{\mathbf{V}_{\mathbf{e}}+\psi \mathbf{V}_{\mathbf{i}}}{1+\psi}$

where $\hat{\mathbf{k}}$ is a unit vector in the direction of observation, i.e. the wavevector direction, and $\psi=v_{e} v_{i} /\left(\Omega_{e} \Omega_{i}\right)$, where $v_{j}$ and $\Omega_{j}$ are the collision and cyclotron frequencies of species $j$ respectively. In the situation contemplated here we have $\mathbf{V}_{e}=\mathbf{V}_{i}=\mathbf{V}_{n}$ so that the structures always move with the plasma along the line-of-sight.

Finally we should recall that as the ions and electrons get lifted by an upward neutral wind, they move above the Es layer equilibrium height. This means that uplifted pieces of Es layers find themselves at a height where the zonal wind moves west on both sides of the layer. As noted above, this tends to make the top side of the uplifted layer more unstable to the gradient-drift instability than the bottom, through the $\mathbf{V}_{z} \times \mathbf{B}$ field. The bottom part could even be stable. Note, however, that since the wind increases with increasing altitude, the destabilizing influence on top of the uplifted layer is larger than the stabilizing influence at the bottom of an uplifted piece. Thus, even in the presence of nonlocal effects associated with the fact that in tilted field lines the unstable and stable regions may be connected via parallel electric fields, a net growth should still be expected. In fact this connection of the unstable region with the stable one may well allow unstable structures to be seen on both sides of the broken Es layer, not to mention inside the uplifted piece of Es layer.

\section{Effects of mean wind fields and ambient electric fields}

Thus far, we have focused on the local agents that make the ions electrons and neutrals move together along the vertical and on the zonal wind origin of the irregularities that allows a radar to see the common vertical motion in the first place. We now briefly consider the possible impact of mean wind fields and ambient electric fields on the observed Doppler shifts and on their impact on the stability of the medium. The discussion is entirely qualitative at this point and only meant as a guide for future research on the question.

\subsection{Impact on observed Doppler shifts and time evolution}

An ambient perpendicular electric field with a strong downward component should be expected during nighttime conditions at low latitudes for two reasons. First, the Gadanki observations are made near the equator, where the electric field is primarily downward at night, owing to the SQ current dynamo. We should also expect in the northern latitudes to get some north-south (nearly downward) electric field from the formation of the Es layer itself: the zonal wind shears should leave some remnant negative charges near the bottom of the layer at its lowest latitudes and some remnant positive charges at its highest latitudes. Such an electric field would match the sign of the vertical Hall field generated in the equatorial electrojet at night.

No matter what its origin might be, a downward/southward component of the electric field creates no Doppler shift in a radar looking up and northward. The only flow triggered by the large scale electric field is associated with an electron $\boldsymbol{E} \times \boldsymbol{B}$ drift. This flow may very well have an impact on the generation of the observed m-size irregularities, but it does not produce an appreciable Doppler shift in either component of the plasma along the electric field direction.

By contrast, an ambient east-west field would certainly be capable of introducing an additional Doppler shift for an observer looking north and up. This can be seen from Eq. (10) which shows that the irregularities should move at a speed that approaches the electron drift along the line of sight. Equations (2) and (3) in turn show that to leading order the ions move very little in the $\boldsymbol{E} \times \boldsymbol{B}$ direction while the opposite is true of electrons. This being stated, the east-west field should be much smaller than the vertical field in and near equatorial regions. Nevertheless steady electron drifts of the order of $10 \mathrm{~m} / \mathrm{s}$ could be present on top of the modulation introduced by the KH billows. This would give the appearance of a mean motion across all altitudes under observation.

We have already discussed the effect of zonal wind shears. On top of the shears, there most likely exists a mean zonal wind. A first consequence of this mean zonal wind is to displace the equilibrium height at which the Es layer will form. A second consequence is to provide a "movie" of the KH billows embedded in the mean flow. A radar looking up to the north and up, perpendicular to the $\mathbf{B}$ direction, can only see time variations in the height and Doppler shift of the billows if these are passing through its field of view. This is clearly the case for the Gadanki observations reported by Choudhary et al. (2005). A second effect of the mean zonal wind is to introduce a very weak ion drift along the vertical. The drift is weak and considered unimportant because the factor $\alpha_{i}$ in Eq. (2) is large.

Finally, there can also be a mean meridional wind. The component of the wind that is parallel to $\mathbf{B}$ (the field is almost horizontal) produces the same effect on ions and electrons and induces a mean Doppler shift. The weaker perpendicular component would induce a small, nearly vertical, mean ion motion. Depending on the east-west extent of the wind field, a measurable mean east-west electric field might or might not be triggered as a result of the ensuing electron $\mathbf{E} \times \mathbf{B}$ drift from the vertical field. An electron Hall drift in the vertical might therefore be present, though a relative drift between ions and electrons may well persist. There may likewise be a shear in the mean meridional wind. This shear 
would introduce a small modulation in the ion drift which would typically not be enough, above $100 \mathrm{~km}$, to cause a reversal in the phase velocity of the structures observed by the radar in view of the prediction from Eq. (10) when $\Psi<1$.

We can conclude that the most important factors are a mean zonal wind, which allows the observer to see the altitude profile of the KH billows with time and a zonal electric field, which would introduce a relatively weak but nevertheless systematic Doppler shift at all altitudes. At night, this should introduce a positive Doppler shift everywhere. In addition, a meridional wind would be seen directly owing to the fact that meridional winds at low latitudes have a strong component along the magnetic field.

\subsection{Impact on the generation of irregularities}

Let $\mathbf{E}_{N}$ be a perpendicular electric field with an upward component (which maps to a northward component at higher latitudes). Aside from the shear in the zonal wind, $\mathbf{E}_{N}$ is the most important factor in the generation of irregularities since this is the component that is in the same direction as the average ambient density gradient. Unlike the sheared zonal wind, however, this ambient electric field only destabilizes one side of the Es layer, namely, the side over which the direction of the electric field has a component parallel to the density gradients. It is not clear, however, if nonlocal effects transmitted along the $\mathbf{B}$ field lines would cancel one another in this case. This would depend largely on the north-south extent of the Es layer pieces. More extended pieces would be more susceptible to nonlocal effects. Still, at very low latitudes, the tilt may be so weak that structures could be triggered on one side of the Es layer by allowing for growth at aspect angles that are within 1 deg of perpendicularity.

An east-west field could actually play a role because of the tilts introduced by the KH billows in the plane perpendicular to the magnetic field (zonal and up). The east-west field could help destabilize the side of the tilted Es layer that would have density gradient component parallel to the eastwest field. Nonlocal effects would not be a problem in this case because the sign of the electric field would not change along a $\mathbf{B}$ line in such a case.

The important destabilizing effects of the zonal wind shears has already been discussed. The additional presence of a mean zonal wind would play a role identical to that of a vertical electric field as far as destabilization is concerned. The stability would therefore depend on whether nonlocal effects are important or not.

The perpendicular component of mean meridional winds would in turn play a role similar to a mean zonal electric field. The effect would be very small, however, because $\mathbf{B}$ is almost horizontal at low latitudes, meaning that the component of the meridional wind in the direction perpendicular to B would be almost negligible.

We conclude that ambient east-west electric fields would be most likely to further destabilize the Es layer once it has been tilted by KH billows. North-south (i.e. almost vertical) electric fields and mean zonal winds might also help destabilize the Es layers. However, in the latter case, the structures would be unstable on only one side of the layer, and nonlocal effects are likely to dampen the net growth through a short-circuiting effect along $\mathbf{B}$ lines.

\section{Summary and conclusion}

Local changes in wind fields should be able to introduce electric fields that are such as to make ions and electrons drift together with the neutrals along certain directions. We have explored this possibility with the fields created by KH billows, based on the observations reported by Choudhary et al. (2005) and based on the simulations by Bernhardt (2002), which produced Es layer tilts during the initial stages of $\mathrm{KH}$ billow evolution. However, we have also shown that in order for irregularities to be present at all, relative drifts between ions and electrons must exist along certain directions. In the $\mathrm{KH}$ billow case this would be the east-west direction, with the primary destabilizing agent most likely to be the shear in the zonal neutral wind. We favor this mechanism because it destabilizes large scale GD waves on both sides of the layers while using the very winds that create an Es layer in the first place. This contrasts with all other possible agents that we have investigated. We have pointed out that in addition to the sheared wind, an ambient east-west field could help destabilize the layers once the KH billows have introduced tilts in the Es layer in the plane perpendicular to the magnetic field direction. We have also noted that mean zonal winds are important as they are the candidate of choice in order for a radar observing in the northward vertical direction to see tilted layers passing through the field of view with time.

Our communication is not necessarily limited to KH billows in that it does suggest that radar observations of plasma irregularities can at times be surprisingly close to the neutral dynamics. However, a careful study of the electrodynamics and of the irregularity generation mechanism is needed on a case-by-case basis even when a gradient-drift instability is excited. Even for the KH billows discussed here, a more detailed study of these questions is warranted, as exemplified by the numerous complications that were raised, but not solved, in Sect. 5.

Acknowledgements. This work has been supported by the Canadian National Science and Engineering Research Council.

Topical Editor M. Pinnock thanks P. A. Bernhardt and an anonymous referee for his help in evaluating this paper.

\section{References}

Bernhardt, P. A.: The modulation of sporadic E layers by KelvinHelmholtz billows in the neutral atmosphere, J. Atmos. Solar Terr. Phys., 64, 1487-1504, 2002. 
Choudhary, R. K., St.-Maurice, J.-P., Kagan, L., and Mahajan, K. K.: Quasi-periodic backscatter from E region at Gadanki: evidence for Kelvin-Helmholtz billows in the lower thermosphere, J. Geophys. Res., 110, doi:10.1029/2004JA010987, 2005.

Cosgrove, R. and Tsunoda, R. T.: A direction-dependent instability of sporadic-E layers in the nighttime midlatitude ionosphere, Geophys. Res. Lett., 29(18), doi:10.1029/2002GL014669, 2002.

Fejer, B. G.: Natural Ionospheric Plasma Waves, in: Modern Ionospheric Science, edited by: Kohl, H., Ruster, R., and Schlegel, K., European Geophysical Society, Katlenburg-Lindau, 216273, 1996.

Gibson-Wilde, D., Werne, J., Fritts, D. C., and Hill, R.: Direct numerical simulation of VHF radar measurements of turbulence in the mesosphere, Radio Sci., 35, 783-798, doi:10.1029/1999RS002269, 2000.

Haldoupis, C. and Schlegel, K.: Characteristics of midlatitude coherent backscatter from the ionospheric $\mathrm{E}$ region obtained with Sporadic E scatter experiment, J. Geophys. Res., 101, $13387-$ 13397, 1996.

Hussey, G. C., Schlegel, K., and Haldoupis, C.: Simultaneous 50$\mathrm{MHz}$ coherent backscatter and digital ionosonde observations in the midlatitude E region, J. Geophys. Res., 103, 6991-7002, doi:10.1029/97JA03089, 1998.
Kagan, L. M. and Kelley, M. C.: A wind driven gradient drift mechanism for mid-latitude E-region ionospheric irregularities, Geophys. Res. Lett., 25, 4141-4144, 1998.

Kelley, M. C.: The Earth's Ionosphere, International Geophysics Series, Academic Press, San Diego, Calif., 487pp, 1989.

Kundu, P. K.: Fluid Mechanics, Academic Press Inc., 2nd edn., 394, 1990.

Larsen, M. F.: A shear instability seeding mechanism for quasiperiodic radar echoes, J. Geophys. Res., 105, 24 931-24 940, 2000.

Riggin, D., Swartz, W. E., Providakes, J., and Farley, D. T.: Radar studies of long-wavelength waves associated with mid-latitude sporadic E layers, J. Geophys. Res., 91, 8011-8024, 1986.

Seyler, C. E., Rosado-Román, J. M., and Farley, D. T.: A nonlocal theory of the gradient-drift instability in the ionospheric E-region plasma at mid-latitudes, J. Atmos. Solar-Terr. Phys., 66, 16271637, doi:10.1016/j.jastp.2004.07.011, 2004.

Whitehead, J. D.: Production and prediction of sporadic E, Rev. Geophys., 8, 65-144, 1970.

Woodman, R. F., Yamamoto, M., and Fukao, S.: Gravity wave modulation of gradient drift instabilities in midlatitude sporadic $\mathrm{E}$ irregularities, Geophys. Res. Lett., 18, 1197-1200, 1991. 